Supplement of Clim. Past, 16, 847-865, 2020

https://doi.org/10.5194/cp-16-847-2020-supplement

(C) Author(s) 2020. This work is distributed under

the Creative Commons Attribution 4.0 License.

(c) (1)

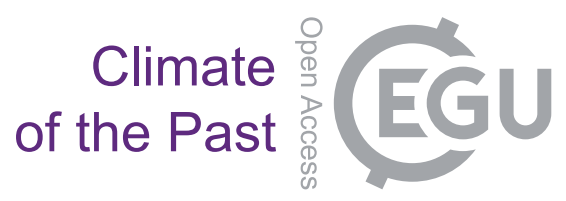

Supplement of

\title{
The origin of Asian monsoons: a modelling perspective
}

Delphine Tardif et al.

Correspondence to: Delphine Tardif (tardif@ipgp.fr)

The copyright of individual parts of the supplement might differ from the CC BY 4.0 License. 


\section{Supplementary Materials}

Figure 1: Wettest month of the year for the control simulation (a) and the GPCP observations. Regions receiving less than $1 \mathrm{~mm} /$ day are kept blank.
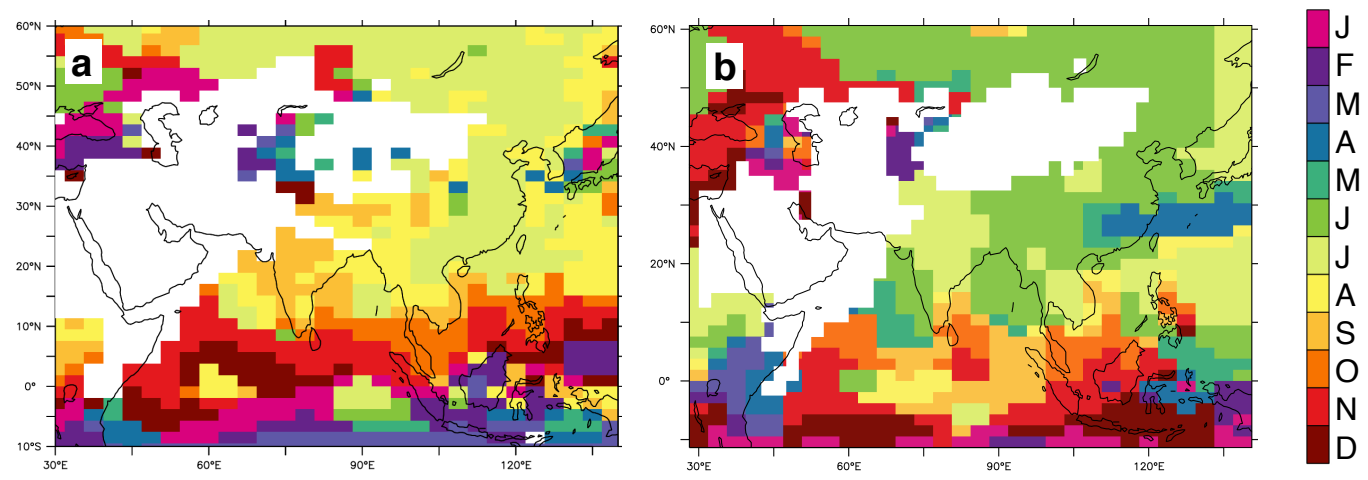

Figure 2: a) paleotopography and paleobathymetry and b) idealized vegetation map prescribed.

For b), color coding is: Boreal Needleleaf Summergreen (red), Boreal Broadleaf Summergreen (cyan), Temperate Broadleaf Evergreen + Temperate Broadleaf Summergreen (light green), Bare soil (yellow), C3 grasses (orange) and Tropical Broadleaved Evergreen + Tropical Broadleaved Raingreen (dark green).
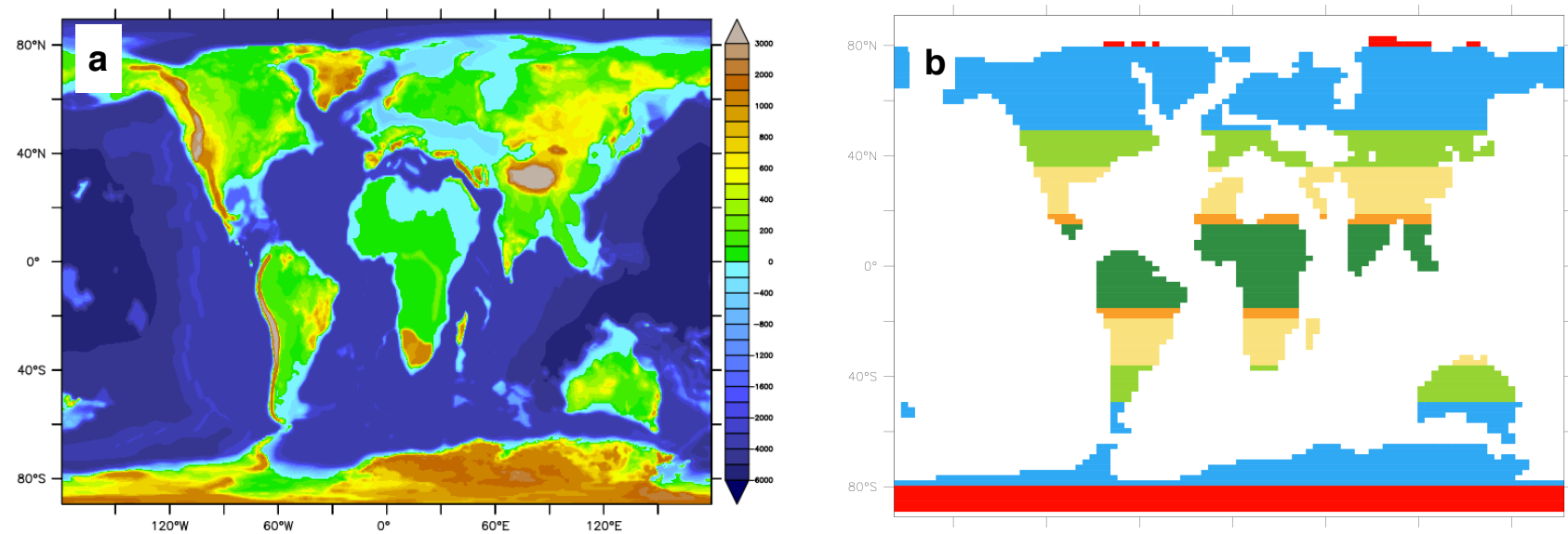
Figure 3: Oceanic heat content stability plots for the 3000 years reference simulations EOC_4X (a). Plotted depth are $5 \mathrm{~m}$ (black), $500 \mathrm{~m}$ (purple), $1800 \mathrm{~m}$ (red) and $4750 \mathrm{~m}$ (blue). The simulation shows a drift inferior to $0.1^{\circ} \mathrm{C}$ per century over the last 500 years for the deep ocean $(4750 \mathrm{~m})$.

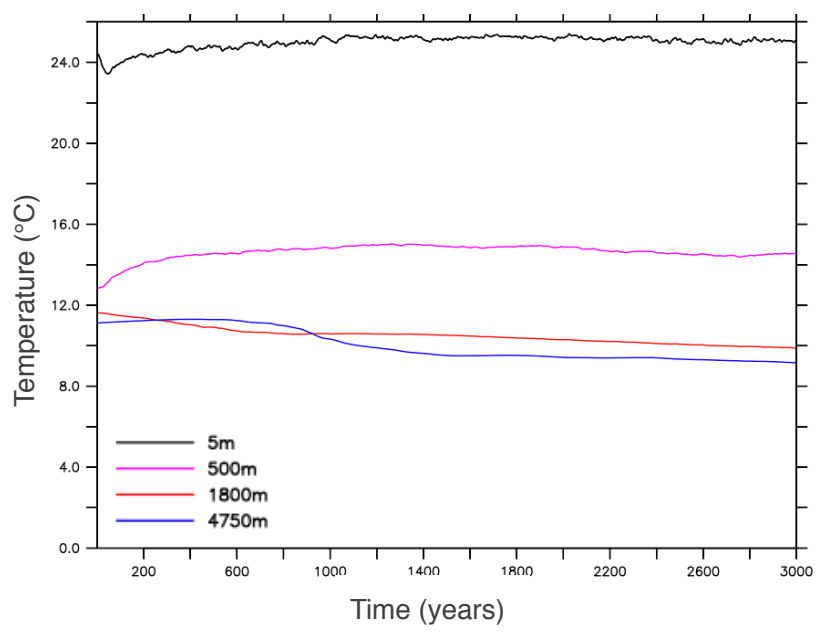

Figure 4: Regression between SST given by the proxies and the one obtained from the model.

In purple: Late-middle Eocene proxy-model regression yields a $\mathbf{r}^{2}=\mathbf{0 . 7 2}$, in orange: late Eocene proxy-model regression a yields $r^{2}=0.54$. The detailed proxy compilation is given in Tables 1 to 4 .

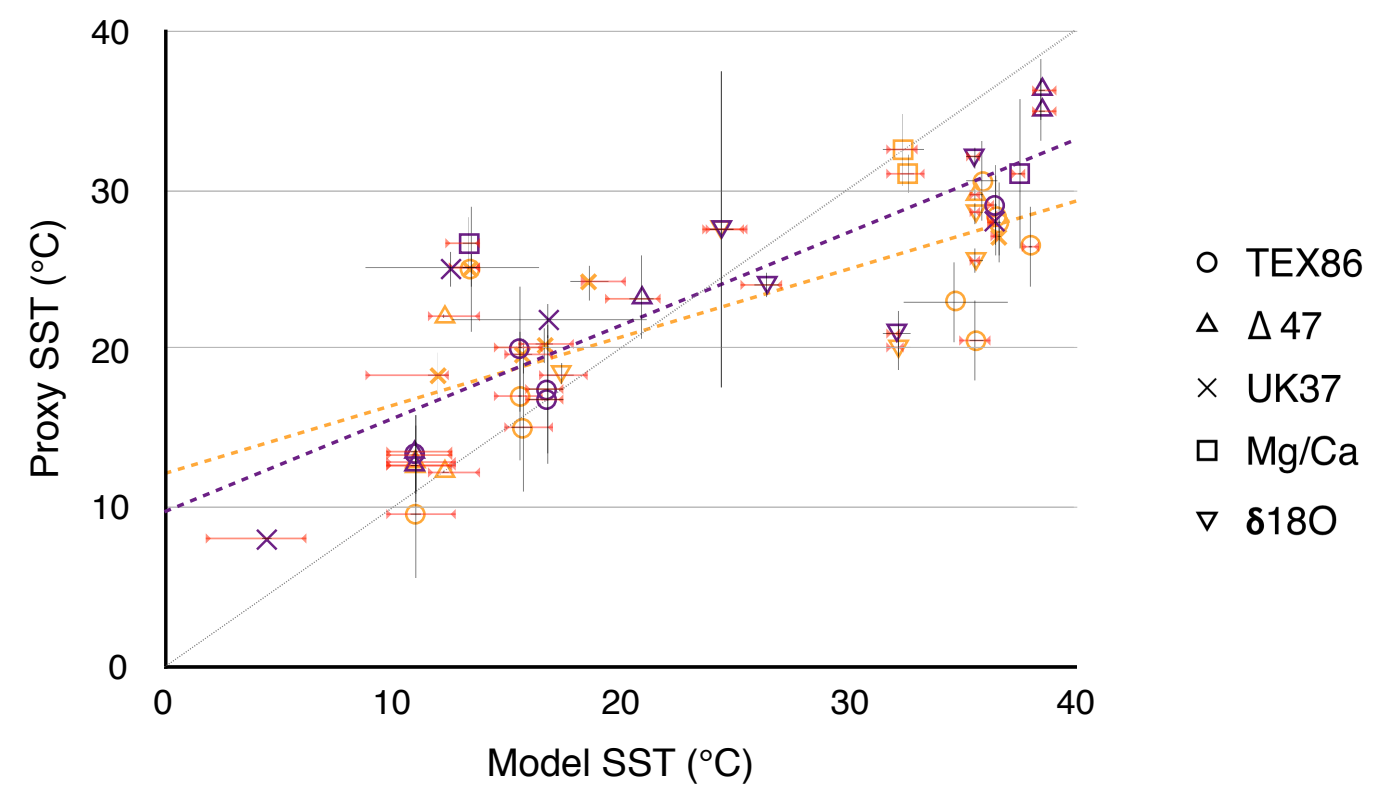


Figure 5: Late Eocene data-model comparison for MAT (a,b), ans SST (c,d). In (a, c), thick line represent the mean temperature from EOC_4X, thin lines are min and max latitudinal temperature from EOC_4X. High altitude proxies $(>1000 \mathrm{~m})$ are represented by triangles, others by circles. In $\mathrm{b}$ pink thick line represents the $10^{\circ} \mathrm{C}$ isotherm. Detailed compilation is provided in Table 1 and 2.
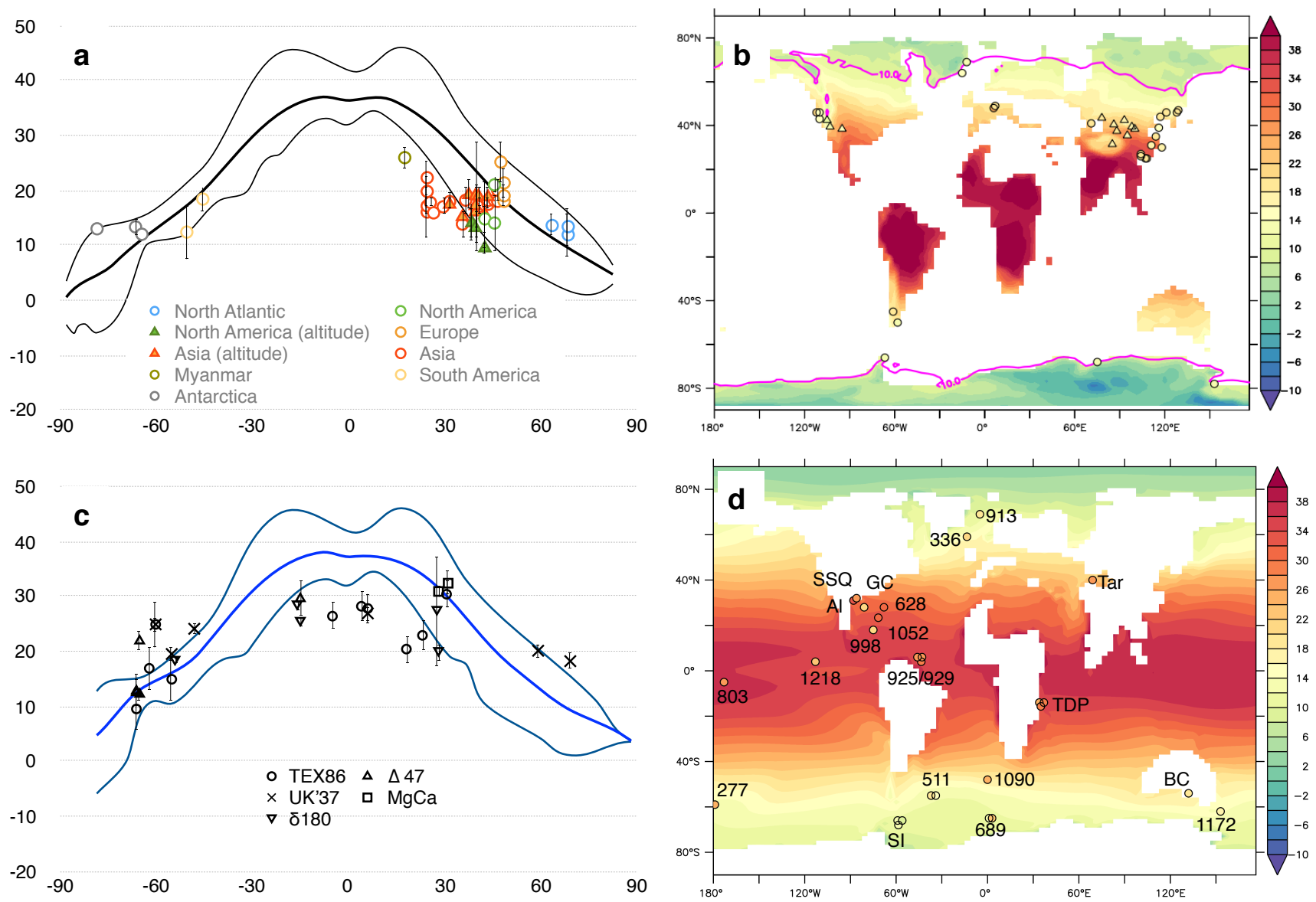


\section{SST and terrestrial temperature compilation description}

The proxy compilations used in this study were taken from an existing compilation by Baatsen et al. (2018). It was further completed with results from the available literature (Hunt et al.2001, Utescher et al. 2015, Kohn et al. 2015, Francis et al. 2007, Greenwood et al. 2010, Licht et al. 2014, Spicer et al. 2017, Hoorn et al. 2012), for the continental compilation. Since a steady declining trend in temperatures is observed through the Middle to Late Eocene (Zachos et al. 2001), samples are divided into two broad categories: «late-middle Eocene» and «late Eocene» (hereafter LME and LE, respectively), corresponding to periods of 42 to $38 \mathrm{Ma}$ and 38 to $34 \mathrm{Ma}$, respectively. As no sharp variation in temperature and $\mathrm{pCO}_{2}$ is expected between LME and LE, some samples, dated around $38 \mathrm{Ma}$, are present in both groups. Considering the scarcity of SST estimates at high northern latitudes, we exceptionally included the $44.5 \mathrm{Ma}$ ACEX drilling SST proxy in the LME group, as no value is available for our period of interest, due to a hiatus in the core spanning from 44.5 to $18.3 \mathrm{Ma}$ (Weller and Stein, 2008). We consider reasonable to include this value in our compilation, keeping in mind that it could present a slight overestimation of the SST at this location.

Note that several TEX 86 calibration methods exist for SST reconstructions. Values from Liu et al. (2009) used the calibration by Kim et al. (2008), while Douglas et al. (2014) used the $\mathrm{TEX}_{86}$ calibration. If studies argued that $\mathrm{TEX}^{\mathrm{L}} 86$ calibration was better adapted to high latitudes (SST below $15^{\circ} \mathrm{C}$ ), and $\mathrm{TEX}^{\mathrm{H}} 86$ for mid to low latitudes, Taylor et al. (2013) showed that was actually more suited for shallow water environments, independently of the SST estimated temperature. Uncertainties remain on this topic, as Ho and Laepple (2016) recently suggested that TEX ${ }^{\mathrm{H}_{86}} \mathrm{might}$ actually reflect subsurface temperatures and therefore challenge the paleoclimatic interpretations based on the usually warm polar SST values measured for the Paleocene or the Eocene periods.

The paleolocation of the proxies was reconstructed using Gplates (www.portal.gplates.org). We differentiate terrestrial proxies results from coastal and low elevation locations from those which paleo-elevation have been estimated higher than $1000 \mathrm{~m}$ (when available). The interest of separating altitude records from other proxies is twofold: first, the coarse resolution of our model induces a smoothing of the elevation, hence a tendency to return higher temperature on the location of high altitude proxies ; second, as fossil material are usually found in basins, they represent the surrounding flora or fauna, whether it comes from the basin area itself or from higher elevation in the surroundings. A fossil assemblage can therefore induce a cold bias on the location of the proxy itself by representing the high altitude neighboring regions. As an elevation difference of $1000 \mathrm{~m}$ for a given latitude is susceptible to induce differences in temperatures of $\sim 6.5^{\circ} \mathrm{C}$, it is an important parameter to consider. 
Table 1: Late Eocene (38-34Ma) terrestrial proxy compilation

\begin{tabular}{|c|c|c|c|c|c|}
\hline Site & Location (paleo) & altitude & references & $\begin{array}{l}\text { MAT } \\
\left({ }^{\circ} \mathrm{C}\right)\end{array}$ & $\begin{array}{l}\text { MAT } \\
\text { error }\end{array}$ \\
\hline \multicolumn{6}{|l|}{ ANTARCTICA } \\
\hline McMurdo & $-77,7^{\circ} \mathrm{N}, 153,2^{\circ} \mathrm{E}$ & $?$ & Passchier et al. (2013) & 13 & 0 \\
\hline King George & $-66^{\circ} \mathrm{N},-66,5^{\circ} \mathrm{E}$ & $?$ & Passchier et al. (2013) & 13 & 2 \\
\hline ODP 1166 & $-68^{\circ} \mathrm{N},-66,5^{\circ} \mathrm{E}$ & $?$ & Passchier et al. (2013) & 12 & 0 \\
\hline \multicolumn{6}{|l|}{ SOUTH AMERICA } \\
\hline Ñirihuau (Chile) & $-45^{\circ} \mathrm{N},-61^{\circ} \mathrm{E}$ & $?$ & Hinojosa \& Villagran (2005) & 18,4 & 0 \\
\hline $\begin{array}{l}\text { Gran Barranca } \\
\text { (Argentina) }\end{array}$ & $-50^{\circ} \mathrm{N},-58^{\circ} \mathrm{E}$ & $?$ & Kohn et al. (2015) & 12,4 & 5 \\
\hline \multicolumn{6}{|l|}{ MYANMAR } \\
\hline Pondaung & $18,5^{\circ} \mathrm{N}, 90,6^{\circ} \mathrm{E}$ & $?$ & Licht et al. (2014) & 26 & 2 \\
\hline \multicolumn{6}{|l|}{ CHINA } \\
\hline $\begin{array}{l}\text { ChangChang (Hainan } \\
\text { Island) }\end{array}$ & $25^{\circ} \mathrm{N}, 107^{\circ} \mathrm{E}$ & No & Spicer et al. (2017) & 22 & 3 \\
\hline $\begin{array}{l}\text { Liushagang I (Leizhou, } \\
\text { Guangdong) }\end{array}$ & $24,6^{\circ} \mathrm{N}, 106,9^{\circ} \mathrm{E}$ & No & Zhang 1981 & 16 & 5 \\
\hline $\begin{array}{l}\text { Youganwo (Maoming } \\
\text { Basin) }\end{array}$ & $25^{\circ} \mathrm{N}, 108^{\circ} \mathrm{E}$ & No & Yu \& Wu 1983 & 17 & 1 \\
\hline $\begin{array}{l}\text { Youganwo (Maoming } \\
\text { Basin) }\end{array}$ & & No & Spicer et al. (2017) & 20 & 3 \\
\hline $\begin{array}{l}\text { Yongning Gr U } \\
\text { (Ningming, Guangxi) }\end{array}$ & $25,5^{\circ} \mathrm{N}, 104,2^{\circ} \mathrm{E}$ & No & Wang 2003 & 18 & 1 \\
\hline Nadu (Baise, Guangxi) & $27,3^{\circ} \mathrm{N}, 103,6^{\circ} \mathrm{E}$ & No & Guo 1979, Liu \& Yang 1999 & 16 & 0 \\
\hline Pinghu (Donghai) & $29,7^{\circ} \mathrm{N}, 119,4^{\circ} \mathrm{E}$ & No & Sun 1989, Zhang 1990 & 17 & 1 \\
\hline Linjiang L (Qingjiang) & $31,6^{\circ} \mathrm{N}, 112,1^{\circ} \mathrm{E}$ & No & He \& Sun 1977 & 18 & 1 \\
\hline $\begin{array}{l}\text { Dagzukha (Xigaze, } \\
\text { Tibet) }\end{array}$ & $32^{\circ} \mathrm{N}, 85,2^{\circ} \mathrm{E}$ & Yes & Li 2009 & 17 & 1 \\
\hline $\begin{array}{l}\text { Dingyuan V (Hefei } \\
\text { Basin) }\end{array}$ & $35,5^{\circ} \mathrm{N}, 114,4^{\circ} \mathrm{E}$ & No & Wang et al 1987 & 14 & 2 \\
\hline $\begin{array}{l}\text { Relu U (Shiqu, } \\
\text { Sichuan) }\end{array}$ & $36,2^{\circ} \mathrm{N}, 94,9^{\circ} \mathrm{E}$ & Yes & Chen 1983 & 15 & 0 \\
\hline $\begin{array}{l}\text { Sanduo U (Gaoyou, } \\
\text { Jiangsu) }\end{array}$ & $36,5^{\circ} \mathrm{N}, 116,6^{\circ} \mathrm{E}$ & No & Zhang \& Qian 1992 & 18 & 3 \\
\hline $\begin{array}{l}\text { Wanbaogou Gr U } \\
\text { (Kulun Pass, Qinghai) }\end{array}$ & $38,1^{\circ} \mathrm{N}, 88,2^{\circ} \mathrm{E}$ & Yes & Zhu 1985 & 19 & 3 \\
\hline Lanzhou, Gansu & $39,4^{\circ} \mathrm{N}, 100,2^{\circ} \mathrm{E}$ & Yes & Ma 1995 & 14 & 2 \\
\hline $\begin{array}{l}\text { Xining Gr (Minhe, } \\
\text { Qinghai) }\end{array}$ & $39,5^{\circ} \mathrm{N}, 100^{\circ} \mathrm{E}$ & Yes & Yu 2003 & 16 & 5 \\
\hline
\end{tabular}




\begin{tabular}{|c|c|c|c|c|c|}
\hline Site & Location (paleo) & altitude & references & $\begin{array}{l}\text { MAT } \\
\left({ }^{\circ} \mathrm{C}\right)\end{array}$ & $\begin{array}{l}\text { MAT } \\
\text { error }\end{array}$ \\
\hline $\begin{array}{l}\text { Honggou III, IV (Xining, } \\
\text { Qinghai) }\end{array}$ & $39,73^{\circ} \mathrm{N}, 98^{\circ} \mathrm{E}$ & Yes & Sun 1980 & 17 & 4 \\
\hline Xining & $40,1^{\circ} \mathrm{N}, 98,7^{\circ} \mathrm{E}$ & Yes & Hoorn, Han & 19 & 10 \\
\hline Xining & & Yes & Wang 1990 & & 0 \\
\hline $\begin{array}{l}\text { Bashibulake (Shache } \\
\text { Basin, Xinjiang) }\end{array}$ & $41^{\circ} \mathrm{N}, 72^{\circ} \mathrm{E}$ & No & Zhao 1982 & 19 & 2 \\
\hline $\begin{array}{l}\text { Xiaganchaigou L } \\
\text { (Mangya, Qinghai) }\end{array}$ & $41,1^{\circ} \mathrm{N}, 86,4^{\circ} \mathrm{E}$ & Yes & Zhu 1985 & 17 & 1 \\
\hline $\begin{array}{l}\text { Huoshaogou Qiaojia } \\
\text { (Yumen, Gansu) }\end{array}$ & $43,4^{\circ} \mathrm{N}, 92,9^{\circ} \mathrm{E}$ & Yes & Miao 2008 & 16 & 6 \\
\hline $\begin{array}{l}\text { Shahejie II } \\
\text { (Qinhuangdao, Hebei) }\end{array}$ & $43,6^{\circ} \mathrm{N}, 116,7^{\circ} \mathrm{E}$ & No & APE \& NIGP 1978 & 17 & 1 \\
\hline $\begin{array}{l}\text { Xiaokuzibai (Kuche } \\
\text { Basin, Xinjiang) }\end{array}$ & $44,1^{\circ} \mathrm{N}, 78,1^{\circ} \mathrm{E}$ & Yes & $\begin{array}{l}\text { Zhao } 1982 \\
\text { Zhang \& Zhan } 1991\end{array}$ & 19 & 2 \\
\hline $\begin{array}{l}\text { Genjiajie (Fushun, } \\
\text { Liaoning) }\end{array}$ & $45,5^{\circ} \mathrm{N}, 121,2^{\circ} \mathrm{E}$ & No & Qu 1993 & 18 & 0 \\
\hline Hunchun & $46^{\circ} \mathrm{N}, 128^{\circ} \mathrm{E}$ & No & Liu 1987, Zhang 1987 & 18 & 0 \\
\hline S Primory'e (Russia) & $47^{\circ} \mathrm{N}, 129^{\circ} \mathrm{E}$ & $?$ & Utescher 2015 & 20 & 2 \\
\hline S Primory'e (Russia) & & $?$ & Utescher 2015 & 18 & 0 \\
\hline \multicolumn{6}{|l|}{ EUROPE } \\
\hline Stare Sedlo & $48^{\circ} \mathrm{N}, 6^{\circ} \mathrm{E}$ & $?$ & Uhl et al, (2007) & 25 & 4 \\
\hline Weiße Elster & $49^{\circ} \mathrm{N}, 6^{\circ} \mathrm{E}$ & $?$ & Uhl et al, (2007) & 21 & 3 \\
\hline $\begin{array}{l}\text { Weiße Elster \& Lausitz } \\
\text { Basin (Knau gravel pit) }\end{array}$ & & $?$ & Utescher 2015 & 18 & 0 \\
\hline $\begin{array}{l}\text { Weiße Elster \& Lausitz } \\
\text { Basin (Haselbach) }\end{array}$ & & $?$ & Utescher 2015 & 19 & 2 \\
\hline \multicolumn{6}{|l|}{ NORTH AMERICA } \\
\hline Florissant CO & $39,25^{\circ} \mathrm{N},-95^{\circ} \mathrm{E}$ & Yes $(3800 \mathrm{~m})$ & $\begin{array}{l}\text { Boyle et al, (2008); Wolfe et al. } \\
\text { (1998) }\end{array}$ & 14 & 3 \\
\hline Sevier (UT) & $40^{\circ} \mathrm{N},-102,9^{\circ} \mathrm{E}$ & $\begin{array}{l}\text { Yes }(3600 \\
+/-700 \mathrm{~m})\end{array}$ & Gregory-Wodzicki (1997) & 13 & 3 \\
\hline Copper Basin (NV) & $43,2^{\circ} \mathrm{N},-105,4^{\circ} \mathrm{E}$ & yes $(2000 m)$ & Wolfe et al, (1998) & 10 & 1 \\
\hline Badger's Nose (CA) & $42,6^{\circ} \mathrm{N},-109,7^{\circ} \mathrm{E}$ & $?$ & Prothero (2008) & 15 & 2 \\
\hline Comstosk (OR) & $45,8^{\circ} \mathrm{N},-112,3^{\circ} \mathrm{E}$ & No & Retallack et al, (2004) & 21 & 1 \\
\hline Gray Butte (OR) & $45,6^{\circ} \mathrm{N},-110,2^{\circ} \mathrm{E}$ & $?$ & Smith et al, (1998) & 14 & 5 \\
\hline
\end{tabular}




\begin{tabular}{|c|c|c|c|c|c|}
\hline Site & Location (paleo) & altitude & references & $\begin{array}{l}\text { MAT } \\
\left({ }^{\circ} \mathrm{C}\right)\end{array}$ & $\begin{array}{l}\text { MAT } \\
\text { error }\end{array}$ \\
\hline \multicolumn{6}{|c|}{ NORTH ATLANTIC } \\
\hline ODP 913 MBT & $69^{\circ} \mathrm{N},-12^{\circ} \mathrm{E}$ & $?$ & Schouten et al, (2008) & 12 & 4 \\
\hline ODP 913 Pollen & & $?$ & Eldrett et al, (2009) & 13 & 3 \\
\hline ODP 643 & $64^{\circ} \mathrm{N},-15^{\circ} \mathrm{E}$ & $?$ & Eldrett et al, (2009) & 14 & 2 \\
\hline
\end{tabular}

Table 2: Late Eocene (38-34 Ma) SST proxy compilation

\begin{tabular}{|c|c|c|c|c|c|}
\hline Site & Location (paleo) & reference & SST $\left({ }^{\circ} \mathrm{C}\right)$ & SST error & Method \\
\hline Seymour Island & $-65.7^{\circ} \mathrm{N},-58^{\circ} \mathrm{E}$ & Douglas et al, (2014) & 12,6 & 2,0 & $\begin{array}{c}\Delta_{47} \\
\text { Cucullaea }\end{array}$ \\
\hline Seymour Island & & Douglas et al, (2014) & 12,9 & 3,0 & $\begin{array}{c}\Delta_{47} \\
\text { Eurhomalea }\end{array}$ \\
\hline Seymour Island & & Douglas et al, (2014) & 9,6 & 4,0 & $\mathrm{TEX}^{\mathrm{L}_{86}}$ \\
\hline $\begin{array}{l}\text { ODP } 689 \\
\text { (Weddell Sea) }\end{array}$ & $-64.7^{\circ} \mathrm{N}, 1.2^{\circ} \mathrm{E}$ & $\begin{array}{l}\text { Petersen and Schrag } \\
\text { (2015) }\end{array}$ & 12,3 & 1,0 & $\Delta 47$ \\
\hline $\begin{array}{l}\text { ODP } 689 \\
\text { (Weddell Sea) }\end{array}$ & & $\begin{array}{l}\text { Petersen and Schrag } \\
(2015)\end{array}$ & 22 & 1,5 & $\Delta 47$ \\
\hline $\begin{array}{l}\text { ODP } 1172 \\
\text { (Tasmania) }\end{array}$ & $-62.4^{\circ} \mathrm{N}, 152.8^{\circ} \mathrm{E}$ & Douglas et al, (2014) & 17 & 4,0 & $\mathrm{TEX}_{86}$ \\
\hline DSDP 277 & $-59.8^{\circ} \mathrm{N}, 179.3^{\circ} \mathrm{E}$ & Douglas et al, (2014) & 25 & 4,0 & $\mathrm{TEX}_{86}$ \\
\hline DSDP 277 & & Liu et al, (2009) & 25 & 1,1 & $\mathrm{UK}_{37}$ \\
\hline DSDP 511 & $-54.9^{\circ} \mathrm{N},-34.1^{\circ} \mathrm{E}$ & Douglas et al, (2014) & 15 & 4,0 & $\mathrm{TEX}^{\mathrm{L}} 86$ \\
\hline DSDP 511 & & Liu et al, (2009) & 19,6 & 1,1 & $\mathrm{UK}^{\mathrm{K}_{37}}$ \\
\hline Brown Creek, Aus & $-54^{\circ} \mathrm{N}, 132^{\circ} \mathrm{E}$ & Kamp et al, (1990) & 18,4 & 0,7 & $\delta^{18} \mathrm{O}$ \\
\hline ODP 1090 & $-47.8^{\circ} \mathrm{N}, 0.2^{\circ} \mathrm{E}$ & Liu et al, (2009) & 24,2 & 1,1 & $\mathrm{UK}_{37}$ \\
\hline Tanzania & $-14.7^{\circ} \mathrm{N}, 33.9^{\circ} \mathrm{E}$ & Pearson et al, (2001) & 25,5 & 0,7 & $\delta^{18} \mathrm{O}$ \\
\hline Tanzania & & Evans et al, (2018) & 29,7 & 3,2 & $\Delta 47$ \\
\hline Tanzania Lindi & $-15.7^{\circ} \mathrm{N}, 34.3^{\circ} \mathrm{E}$ & Pearson et al, (2001) & 28,5 & 0,7 & $\delta^{18} \mathrm{O}$ \\
\hline ODP 929 & $-5.9^{\circ} \mathrm{N},-43.5^{\circ} \mathrm{E}$ & Liu et al, (2009) & 27,9 & 2,5 & $\mathrm{TEX}_{86}$ \\
\hline ODP 929 & & Liu et al, (2009) & 27 & 1,1 & $\mathrm{UK}^{\prime}{ }_{37}$ \\
\hline ODP 803 & $-5.2^{\circ} \mathrm{N},-172.8^{\circ} \mathrm{E}$ & Liu et al, (2009) & 26,5 & 2,5 & TEX $_{86}$ \\
\hline ODP 1218 & $4^{\circ} \mathrm{N},-113.5^{\circ} \mathrm{E}$ & Liu et al, (2009) & 23 & 2,5 & $\mathrm{TEX}_{86}$ \\
\hline ODP 925 & $4.2^{\circ} \mathrm{N},-43.5^{\circ} \mathrm{E}$ & Liu et al, (2009) & 28,3 & 2,5 & TEX $_{86}$ \\
\hline ODP 998 & $18.1^{\circ} \mathrm{N},-75^{\circ} \mathrm{E}$ & Liu et al, (2009) & 20,5 & 2,5 & $\mathrm{TEX}_{86}$ \\
\hline ODP 628 & $23.4^{\circ} \mathrm{N},-71.7^{\circ} \mathrm{E}$ & Liu et al, (2009) & 30,5 & 2,5 & $\mathrm{TEX}_{86}$ \\
\hline ODP 1052 & $28^{\circ} \mathrm{N},-67.9^{\circ} \mathrm{E}$ & Okafor et al, (2009) & 31 & 1,2 & $\mathrm{Mg} / \mathrm{Ca}$ \\
\hline Gulf Coast, USA & $28.4^{\circ} \mathrm{N},-81.1^{\circ} \mathrm{E}$ & Kobashi et al, (2004) & 20 & & $\delta^{18} \mathrm{O}$ \\
\hline
\end{tabular}




\begin{tabular}{|l|c|c|cc|c|}
\hline Site & Location (paleo) & reference & SST $\left({ }^{\circ} \mathrm{C}\right)$ & SST error & Method \\
\hline St Stephens Quarry & $31^{\circ} \mathrm{N},-87.8^{\circ} \mathrm{E}$ & Wade et al, (2012) & 32,5 & 2,2 & $\mathrm{Mg} / \mathrm{Ca}$ \\
\hline Alabama US & $31.5^{\circ} \mathrm{N},-88^{\circ} \mathrm{E}$ & Pearson et al, (2001) & 26,5 & 0,7 & $\delta^{18} \mathrm{O}$ \\
\hline Tarim & $40^{\circ} \mathrm{N}, 75^{\circ} \mathrm{E}$ & Bougeois et al. (2016) & 27,5 & 10 & $\delta^{18} \mathrm{O}$ \\
\hline DSDP 336 & $59.1^{\circ} \mathrm{N},-13.5^{\circ} \mathrm{E}$ & Liu et al, (2009) & 20,2 & 1,1 & $\mathrm{UK}^{\prime} 37$ \\
\hline ODP 913 & $69^{\circ} \mathrm{N},-5^{\circ} \mathrm{E}$ & Liu et al, (2009) & 18,3 & 1,1 & $\mathrm{UK}^{\mathrm{K}_{37}}$ \\
\hline
\end{tabular}

Table 3: Late middle Eocene (42-38Ma) terrestrial proxy compilation

\begin{tabular}{|c|c|c|c|c|c|}
\hline Site & Location (paleo) & altitude & references & $\begin{array}{r}\text { MAT } \\
\left({ }^{\circ} \mathrm{C}\right)\end{array}$ & $\begin{array}{l}\text { MAT } \\
\text { error }\end{array}$ \\
\hline \multicolumn{6}{|l|}{ ANTARCTICA } \\
\hline McMurdo & $-77,7^{\circ} \mathrm{N}, 153,2^{\circ} \mathrm{E}$ & $?$ & Passchier et al. (2013) & 13 & 0 \\
\hline King George & $-66^{\circ} \mathrm{N},-66,5^{\circ} \mathrm{E}$ & $?$ & Passchier et al. (2013) & 13 & 2 \\
\hline ODP 1166 & $-68^{\circ} \mathrm{N},-66,5^{\circ} \mathrm{E}$ & $?$ & Passchier et al. (2013) & 12 & 0 \\
\hline \multicolumn{6}{|l|}{ SOUTH AMERICA } \\
\hline Ñirihuau (Chile) & $-45^{\circ} \mathrm{N},-61^{\circ} \mathrm{E}$ & $?$ & Hinojosa \& Villagran (2005) & 18,4 & 0 \\
\hline $\begin{array}{l}\text { Gran Barranca } \\
\text { (Argentina) }\end{array}$ & $-50^{\circ} \mathrm{N},-58^{\circ} \mathrm{E}$ & $?$ & Kohn et al. (2015) & 12,4 & 5 \\
\hline \multicolumn{6}{|l|}{ MYANMAR } \\
\hline Pondaung & $18,5^{\circ} \mathrm{N}, 90,6^{\circ} \mathrm{E}$ & $?$ & Licht et al. (2014) & 26 & 2 \\
\hline \multicolumn{6}{|l|}{ ASIA } \\
\hline $\begin{array}{l}\text { ChangChang (Hainan } \\
\text { Island) }\end{array}$ & $25^{\circ} \mathrm{N}, 107^{\circ} \mathrm{E}$ & No & Spicer et al. (2017) & 22 & 3 \\
\hline $\begin{array}{l}\text { Liushagang I (Leizhou, } \\
\text { Guangdong) }\end{array}$ & $24,6^{\circ} \mathrm{N}, 106,9^{\circ} \mathrm{E}$ & No & Zhang 1981 & 16 & 5 \\
\hline $\begin{array}{l}\text { Youganwo (Maoming } \\
\text { Basin) }\end{array}$ & $25^{\circ} \mathrm{N}, 108^{\circ} \mathrm{E}$ & No & Yu \& Wu 1983 & 17 & 1 \\
\hline $\begin{array}{l}\text { Youganwo (Maoming } \\
\text { Basin) }\end{array}$ & & No & Spicer et al. (2017) & 20 & 3 \\
\hline $\begin{array}{l}\text { Yongning Gr U } \\
\text { (Ningming, Guangxi) }\end{array}$ & $25,5^{\circ} \mathrm{N}, 104,2^{\circ} \mathrm{E}$ & No & Wang 2003 & 18 & 1 \\
\hline Nadu (Baise, Guangxi) & $27,3^{\circ} \mathrm{N}, 103,6^{\circ} \mathrm{E}$ & No & Guo 1979, Liu \& Yang 1999 & 16 & 0 \\
\hline Pinghu (Donghai) & $29,7^{\circ} \mathrm{N}, 119,4^{\circ} \mathrm{E}$ & No & Sun 1989, Zhang 1990 & 17 & 1 \\
\hline Linjiang L (Qingjiang) & $31,6^{\circ} \mathrm{N}, 112,1^{\circ} \mathrm{E}$ & No & He \& Sun 1977 & 18 & 1 \\
\hline $\begin{array}{l}\text { Dagzukha (Xigaze, } \\
\text { Tibet) }\end{array}$ & $32^{\circ} \mathrm{N}, 85,2^{\circ} \mathrm{E}$ & Yes & Li 2009 & 17 & 1 \\
\hline Jianghan Basin & $34^{\circ} \mathrm{N}, 111^{\circ} \mathrm{E}$ & $?$ & Ma 2012 & 20 & 2 \\
\hline
\end{tabular}




\begin{tabular}{|c|c|c|c|c|c|}
\hline Site & Location (paleo) & altitude & references & $\begin{array}{r}\text { MAT } \\
\left({ }^{\circ} \mathrm{C}\right)\end{array}$ & $\begin{array}{l}\text { MAT } \\
\text { error }\end{array}$ \\
\hline $\begin{array}{l}\text { Dingyuan V (Hefei } \\
\text { Basin) }\end{array}$ & $35,5^{\circ} \mathrm{N}, 114,4^{\circ} \mathrm{E}$ & No & Wang et al 1987 & 14 & 2 \\
\hline Cheshme & $36^{\circ} \mathrm{N}, 61^{\circ} \mathrm{E}$ & $?$ & Akhmetiev 2014 & & \\
\hline $\begin{array}{l}\text { Relu U (Shiqu, } \\
\text { Sichuan) }\end{array}$ & $36,2^{\circ} \mathrm{N}, 94,9^{\circ} \mathrm{E}$ & Yes & Chen 1983 & 15 & 0 \\
\hline $\begin{array}{l}\text { Sanduo U (Gaoyou, } \\
\text { Jiangsu) }\end{array}$ & $36,5^{\circ} \mathrm{N}, 116,6^{\circ} \mathrm{E}$ & No & Zhang \& Qian 1992 & 18 & 3 \\
\hline $\begin{array}{l}\text { Wanbaogou Gr U } \\
\text { (Kulun Pass, Qinghai) }\end{array}$ & $38,1^{\circ} \mathrm{N}, 88,2^{\circ} \mathrm{E}$ & Yes & Zhu 1985 & 19 & 3 \\
\hline Lanzhou, Gansu & $39,4^{\circ} \mathrm{N}, 100,2^{\circ} \mathrm{E}$ & Yes & Ma 1995 & 14 & 2 \\
\hline $\begin{array}{l}\text { Xining Gr (Minhe, } \\
\text { Qinghai) }\end{array}$ & $39,5^{\circ} \mathrm{N}, 100^{\circ} \mathrm{E}$ & Yes & Yu 2003 & 16 & 5 \\
\hline $\begin{array}{l}\text { Honggou III, IV (Xining, } \\
\text { Qinghai) }\end{array}$ & $39,73^{\circ} \mathrm{N}, 98^{\circ} \mathrm{E}$ & Yes & Sun 1980 & 17 & 4 \\
\hline Xining & $40,1^{\circ} \mathrm{N}, 98,7^{\circ} \mathrm{E}$ & Yes & Hoorn, Han & 19 & 10 \\
\hline $\begin{array}{l}\text { Bashibulake (Shache } \\
\text { Basin, Xinjiang) }\end{array}$ & $41^{\circ} \mathrm{N}, 72^{\circ} \mathrm{E}$ & No & Zhao 1982 & 19 & 2 \\
\hline $\begin{array}{l}\text { Xiaganchaigou L } \\
\text { (Mangya, Qinghai) }\end{array}$ & $41,1^{\circ} \mathrm{N}, 86,4^{\circ} \mathrm{E}$ & Yes & Zhu 1985 & 17 & 1 \\
\hline $\begin{array}{l}\text { Huoshaogou Qiaojia } \\
\text { (Yumen, Gansu) }\end{array}$ & $43,4^{\circ} \mathrm{N}, 92,9^{\circ} \mathrm{E}$ & Yes & Miao 2008 & 16 & 6 \\
\hline $\begin{array}{l}\text { Shahejie II } \\
\text { (Qinhuangdao, Hebei) }\end{array}$ & $43,6^{\circ} \mathrm{N}, 116,7^{\circ} \mathrm{E}$ & No & APE \& NIGP 1978 & 17 & 1 \\
\hline $\begin{array}{l}\text { Xiaokuzibai (Kuche } \\
\text { Basin, Xinjiang) }\end{array}$ & $44,1^{\circ} \mathrm{N}, 78,1^{\circ} \mathrm{E}$ & Yes & $\begin{array}{l}\text { Zhao } 1982 \\
\text { Zhang \& Zhan } 1991\end{array}$ & 19 & 2 \\
\hline $\begin{array}{l}\text { Genjiajie (Fushun, } \\
\text { Liaoning) }\end{array}$ & $45,5^{\circ} \mathrm{N}, 121,2^{\circ} \mathrm{E}$ & No & Qu 1993 & 18 & 0 \\
\hline Fushun, Liaoning & $45,5^{\circ} \mathrm{N}, 121,2^{\circ} \mathrm{E}$ & No & Ma 2012 & 18 & 2 \\
\hline Hunchun & $46^{\circ} \mathrm{N}, 128^{\circ} \mathrm{E}$ & No & Liu 1987, Zhang 1987 & 18 & 0 \\
\hline S Primorye (Russia) & $47^{\circ} \mathrm{N}, 129^{\circ} \mathrm{E}$ & $?$ & Utescher 2015 & 20 & 2 \\
\hline S Primorye (Russia) & & $?$ & Utescher 2015 & 18 & 0 \\
\hline S Primorye & & $?$ & Akhmetiev 2014 & & \\
\hline Huadian Basin & $47,7^{\circ} \mathrm{N}, 124^{\circ} \mathrm{E}$ & No & Ma 2012 & 17 & 1 \\
\hline Zaisan Basin & $48^{\circ} \mathrm{N}, 85^{\circ} \mathrm{E}$ & $?$ & Akhmetiev 2014 & & \\
\hline Yilan-Yitong & $50.5^{\circ} \mathrm{N}, 126^{\circ} \mathrm{E}$ & $?$ & Ma 2012 & & \\
\hline Turgai & $51^{\circ} \mathrm{N}, 57^{\circ} \mathrm{E}$ & $?$ & Akhmetiev 2014 & & \\
\hline Pavlodar & $54^{\circ} \mathrm{N}, 70^{\circ} \mathrm{E}$ & $?$ & Akhmetiev 2014 & & \\
\hline$=$ & & & & & \\
\hline
\end{tabular}




\begin{tabular}{|c|c|c|c|c|c|}
\hline Site & Location (paleo) & altitude & references & $\begin{array}{l}\text { MAT } \\
\left({ }^{\circ} \mathrm{C}\right)\end{array}$ & $\begin{array}{l}\text { MAT } \\
\text { error }\end{array}$ \\
\hline Stare Sedlo & $48^{\circ} \mathrm{N}, 6^{\circ} \mathrm{E}$ & $?$ & Uhl et al, (2007) & 25 & 4 \\
\hline Weiße Elster & $49^{\circ} \mathrm{N}, 6^{\circ} \mathrm{E}$ & $?$ & Uhl et al, (2007) & 21 & 3 \\
\hline $\begin{array}{l}\text { Weiße Elster \& Lausitz } \\
\text { Basin (Knau gravel pit) }\end{array}$ & & $?$ & Utescher 2015 & 18 & 0 \\
\hline $\begin{array}{l}\text { Weiße Elster \& Lausitz } \\
\text { Basin (Haselbach) }\end{array}$ & & $?$ & Utescher 2015 & 19 & 2 \\
\hline \multicolumn{6}{|l|}{ NORTH AMERICA } \\
\hline Florissant CO & $39,25^{\circ} \mathrm{N},-95^{\circ} \mathrm{E}$ & $\begin{array}{c}\text { Yes } \\
(3800 \mathrm{~m})\end{array}$ & $\begin{array}{l}\text { Boyle et al, (2008) ; Wolfe et } \\
\text { al. (1998) }\end{array}$ & 14 & 3 \\
\hline Sevier (UT) & $40^{\circ} \mathrm{N},-102,9^{\circ} \mathrm{E}$ & $\begin{array}{l}\text { Yes }(3600 \\
+/-700 \mathrm{~m})\end{array}$ & Gregory-Wodzicki (1997) & 13 & 3 \\
\hline Copper Basin (NV) & $43,2^{\circ} \mathrm{N},-105,4^{\circ} \mathrm{E}$ & $\begin{array}{c}\text { yes } \\
(2000 \mathrm{~m})\end{array}$ & Wolfe et al, (1998) & 10 & 1 \\
\hline Badger's Nose (CA) & $42,6^{\circ} \mathrm{N},-109,7^{\circ} \mathrm{E}$ & $?$ & Prothero (2008) & 15 & 2 \\
\hline Comstosk (OR) & $45,8^{\circ} \mathrm{N},-112,3^{\circ} \mathrm{E}$ & No & Retallack et al, (2004) & 21 & 1 \\
\hline Gray Butte (OR) & $45,6^{\circ} \mathrm{N},-110,2^{\circ} \mathrm{E}$ & $?$ & Smith et al, (1998) & 14 & 5 \\
\hline \multicolumn{6}{|l|}{ NORTH ATLANTIC } \\
\hline ODP 913 MBT & $69^{\circ} \mathrm{N},-12^{\circ} \mathrm{E}$ & $?$ & Schouten et al, (2008) & 12 & 4 \\
\hline ODP 913 Pollen & & $?$ & Eldrett et al, (2009) & 13 & 3 \\
\hline ODP 643 & $64^{\circ} \mathrm{N},-15^{\circ} \mathrm{E}$ & $?$ & Eldrett et al, (2009) & 14 & 2 \\
\hline
\end{tabular}

Table 4: Late Middle Eocene (42-38 Ma) SST proxy compilation

\begin{tabular}{|c|c|c|c|c|c|}
\hline Site & Location (paleo) & reference & SST $\left({ }^{\circ} \mathrm{C}\right)$ & SST error & Method \\
\hline Seymour Island & $-65.7^{\circ} \mathrm{N},-58^{\circ} \mathrm{E}$ & Douglas et al. (2014) & 12,6 & 2,4 & $\begin{array}{c}\Delta_{47} \\
\text { Cucullaea }\end{array}$ \\
\hline Seymour Island & & Douglas et al. (2014) & 13,5 & 2,0 & $\begin{array}{c}\Delta_{47} \\
\text { Eurhomalea }\end{array}$ \\
\hline Seymour Island & & Douglas et al. (2014) & 13,4 & 4,0 & $\mathrm{TEX}^{\mathrm{L}} 86$ \\
\hline ODP 1172 & $-62.4^{\circ} \mathrm{N}, 152.8^{\circ} \mathrm{E}$ & $\begin{array}{l}\text { Bijl et al, (2009), values with } \\
\text { TEX' }_{86} \text { calibration in } \\
\text { Douglas et al. (2014) }\end{array}$ & 20 & 4,0 & $\mathrm{TEX}^{\mathrm{L}} 86$ \\
\hline DSDP 277 & $-59.8^{\circ} \mathrm{N}, 179.3^{\circ} \mathrm{E}$ & Hines et al, (2017) & 26,6 & 1,7 & $\mathrm{Mg} / \mathrm{Ca}$ \\
\hline $\begin{array}{l}\text { New Zealand } \\
\text { (Hampden) }\end{array}$ & $-51^{\circ} \mathrm{N},-165^{\circ} \mathrm{E}$ & Hollis et al. (2012) & 16,8 & 4,0 & $\mathrm{TEX}^{\mathrm{L}} 86$ \\
\hline $\begin{array}{l}\text { New Zealand } \\
\text { (Mid Waipara) }\end{array}$ & & Hollis et al. (2012) & 17,4 & 4,0 & $\mathrm{TEX}_{86}$ \\
\hline
\end{tabular}




\begin{tabular}{|c|c|c|c|c|c|}
\hline Site & Location (paleo) & reference & SST $\left({ }^{\circ} \mathrm{C}\right)$ & SST error & Method \\
\hline Tanzania Lindi & $-15.7^{\circ} \mathrm{N}, 34.3^{\circ} \mathrm{E}$ & Pearson et al, (2001) & 32 & 0,7 & $\delta^{18} \mathrm{O}$ \\
\hline Java KW01 & $-0.4^{\circ} \mathrm{N}, 109.2^{\circ} \mathrm{E}$ & Evans et al, (2018) & 35 & 2,0 & $\Delta_{47}$ \\
\hline Java KW01 & & Evans et al, (2018) & 36,3 & 1,9 & $\Delta_{47}$ \\
\hline ODP 925 & $1.4^{\circ} \mathrm{N},-151.4^{\circ} \mathrm{E}$ & Liu et al, (2009) & 28 & 1,1 & $\mathrm{UK}_{37}^{\prime}$ \\
\hline ODP 925 & & Liu et al, (2009) & 29 & 2,5 & $\mathrm{TEX}_{86}$ \\
\hline ODP 865 & $8.3^{\circ} \mathrm{N},-151.4^{\circ} \mathrm{E}$ & Tripati et al, (2003) & 31 & 4,7 & $\mathrm{Mg} / \mathrm{Ca}$ \\
\hline Gulf Coast, USA & $28.4^{\circ} \mathrm{N},-81.1^{\circ} \mathrm{E}$ & Kobashi et al, (2004) & 21 & 0,7 & $\delta^{18} \mathrm{O}$ \\
\hline Istra More 5 & $39^{\circ} \mathrm{N}, 10^{\circ} \mathrm{E}$ & Pearson et al, (2001) & 24 & 0,7 & $\delta^{18} \mathrm{O}$ \\
\hline Tarim & $40^{\circ} \mathrm{N}, 77^{\circ} \mathrm{E}$ & Bougeois 2016 & 27,5 & 10 & $\delta^{18} \mathrm{O}$ \\
\hline Hampshire Basin & $48.4^{\circ} \mathrm{N},-6.4^{\circ} \mathrm{E}$ & Evans et al, (2018) & 23,2 & 2,6 & $\Delta_{47}$ \\
\hline DSDP 336 & $59.1^{\circ} \mathrm{N},-13.5^{\circ} \mathrm{E}$ & Liu et al, (2009) & 21,8 & 1,1 & $\mathrm{UK}^{\prime}{ }_{37}$ \\
\hline ODP 913 & $69^{\circ} \mathrm{N},-5^{\circ} \mathrm{E}$ & Liu et al, (2009) & 25 & 1,1 & $\mathrm{UK}^{\mathrm{K}_{37}}$ \\
\hline ACEX & $87.9^{\circ} \mathrm{N}, 136.2^{\circ} \mathrm{E}$ & Weller and Stein 2007 & 8 & 1,1 & $U^{K^{\prime}}{ }_{37}$ \\
\hline
\end{tabular}

Table 5: Qualitative proxy compilation used in Figure 4a.

\begin{tabular}{|c|c|c|c|}
\hline Site & Location (modern) & reference & $\begin{array}{l}\text { main qualitative } \\
\text { composition }\end{array}$ \\
\hline Yilan-Yitong (China) & $46^{\circ} \mathrm{N}, 129^{\circ} \mathrm{E}$ & Ma et al. 2012 & forest \\
\hline Huadian (China) & $43^{\circ} \mathrm{N}, 127^{\circ} \mathrm{E}$ & Ma et al. 2012 & forest \\
\hline Fushun (China) & $42^{\circ} \mathrm{N}, 124^{\circ} \mathrm{E}$ & Ma et al. 2012 & forest \\
\hline Jiuquan (China) & $40^{\circ} \mathrm{N}, 97^{\circ} \mathrm{E}$ & Ma et al. 2012 & shrub/grass \\
\hline Qaidam (China) & $38^{\circ} \mathrm{N}, 91^{\circ} \mathrm{E}$ & Ma et al. 2012 & shrub/grass \\
\hline Dahonggou (China) & $37^{\circ} \mathrm{N}, 95^{\circ} \mathrm{E}$ & Ma et al. 2012 & shrub/grass \\
\hline Xining (China) & $37^{\circ} \mathrm{N}, 102^{\circ} \mathrm{E}$ & Hoorn et al. 2012 & shrub/grass \\
\hline Lushi (China) & $34^{\circ} \mathrm{N}, 111^{\circ} \mathrm{E}$ & Ma et al. 2012 & grass \\
\hline Tantou (China) & $34^{\circ} \mathrm{N}, 118^{\circ} \mathrm{E}$ & Ma et al. 2012 & forest \\
\hline Tailai (China) & $36^{\circ} \mathrm{N}, 117^{\circ} \mathrm{E}$ & Ma et al. 2012 & forest \\
\hline Nanling (China) & $31^{\circ} \mathrm{N}, 118^{\circ} \mathrm{E}$ & Ma et al. 2012 & forest \\
\hline Jianghan (China) & $30^{\circ} \mathrm{N}, 113^{\circ} \mathrm{E}$ & Ma et al. 2012 & shrub/forest \\
\hline Baise (China) & $22^{\circ} \mathrm{N}, 109^{\circ} \mathrm{E}$ & Ma et al. 2012 & forest \\
\hline Assam (India) & $26^{\circ} \mathrm{N}, 92^{\circ} \mathrm{E}$ & Saxena and Trivedi 2009 & forest \\
\hline southern India & $12^{\circ} \mathrm{N}, 79^{\circ} \mathrm{E}$ & Boucot et al. 2013 & forest \\
\hline Kashmir (India) & $39^{\circ} \mathrm{N}, 75^{\circ} \mathrm{E}$ & Boucot et al. 2013 & forest \\
\hline Cambay (India) & $22^{\circ} \mathrm{N}, 73^{\circ} \mathrm{E}$ & Boucot et al. 2013 & forest \\
\hline
\end{tabular}




\begin{tabular}{|c|c|c|c|}
\hline Rajasthan (India) & $27^{\circ} \mathrm{N}, 74^{\circ} \mathrm{E}$ & Boucot et al. 2013 & forest \\
\hline Pondaung (Myanmar) & $21^{\circ} \mathrm{N}, 94^{\circ} \mathrm{E}$ & Licht et al. 2014 & forest \\
\hline $\begin{array}{l}\text { Nanggulan and Walat } \\
\text { (Java) }\end{array}$ & $6^{\circ} \mathrm{S}, 107^{\circ} \mathrm{E}$ & Morley 2018 & forest \\
\hline Mangkalihat (Kalimantan) & $1^{\circ} \mathrm{N}, 118^{\circ} \mathrm{E}$ & Morley 2018 & forest \\
\hline Turgai (Russia) & $51^{\circ} \mathrm{N}, 57^{\circ} \mathrm{E}$ & $\begin{array}{c}\text { Akhmetiev and } \\
\text { Zaporozhets } 2014\end{array}$ & forest \\
\hline Zaisan (Russia) & $48^{\circ} \mathrm{N}, 85^{\circ} \mathrm{E}$ & $\begin{array}{c}\text { Akhmetiev and } \\
\text { Zaporozhets } 2014\end{array}$ & forest \\
\hline Chesme (Turkmenistan) & $36^{\circ} \mathrm{N}, 61^{\circ} \mathrm{E}$ & $\begin{array}{c}\text { Akhmetiev and } \\
\text { Zaporozhets } 2014\end{array}$ & shrub \\
\hline
\end{tabular}

Figure 6 : JJA Air Temperature (in Kelvin) at 300 mb for Control (a) ERA 5 reanalysis (b) with contours overlaid each degree.
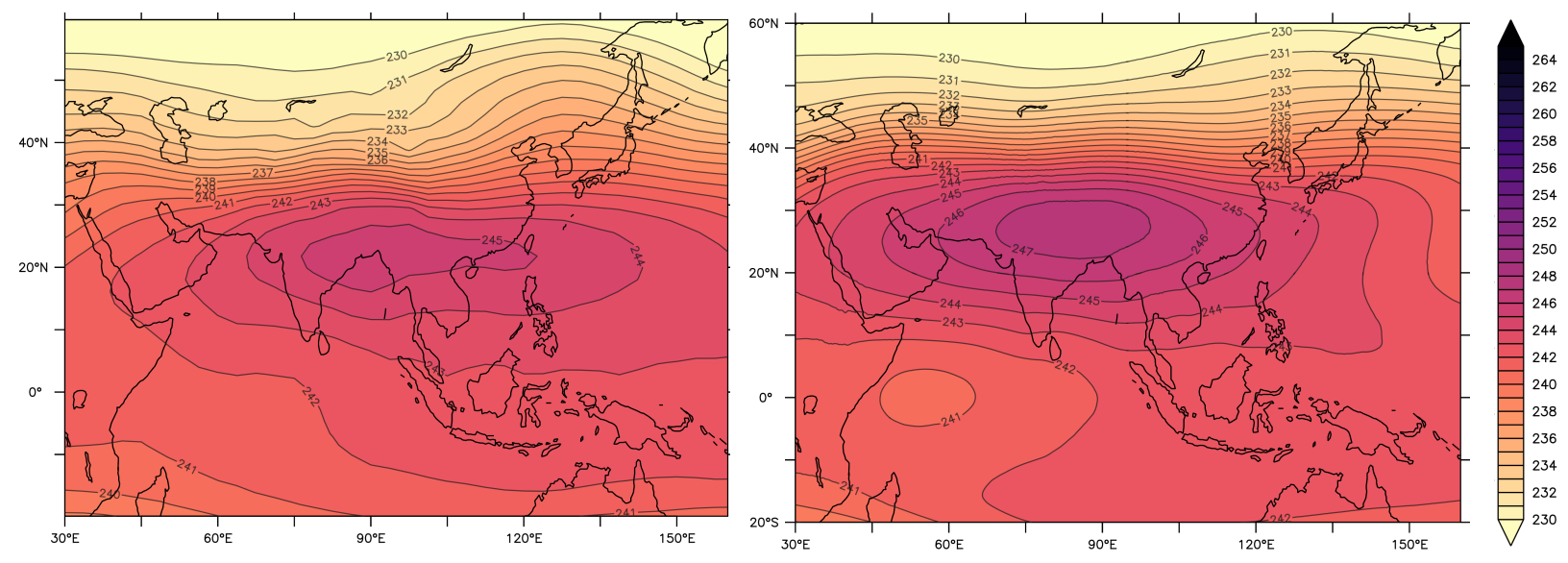
Figure 7 : Late Eocene Mean Annual Precipitations are shaded (in mm/year) and compared to the occurrence of arid climate related evaporites deposits (red diamonds) and more ever-wet climate related coal deposits (green circles).

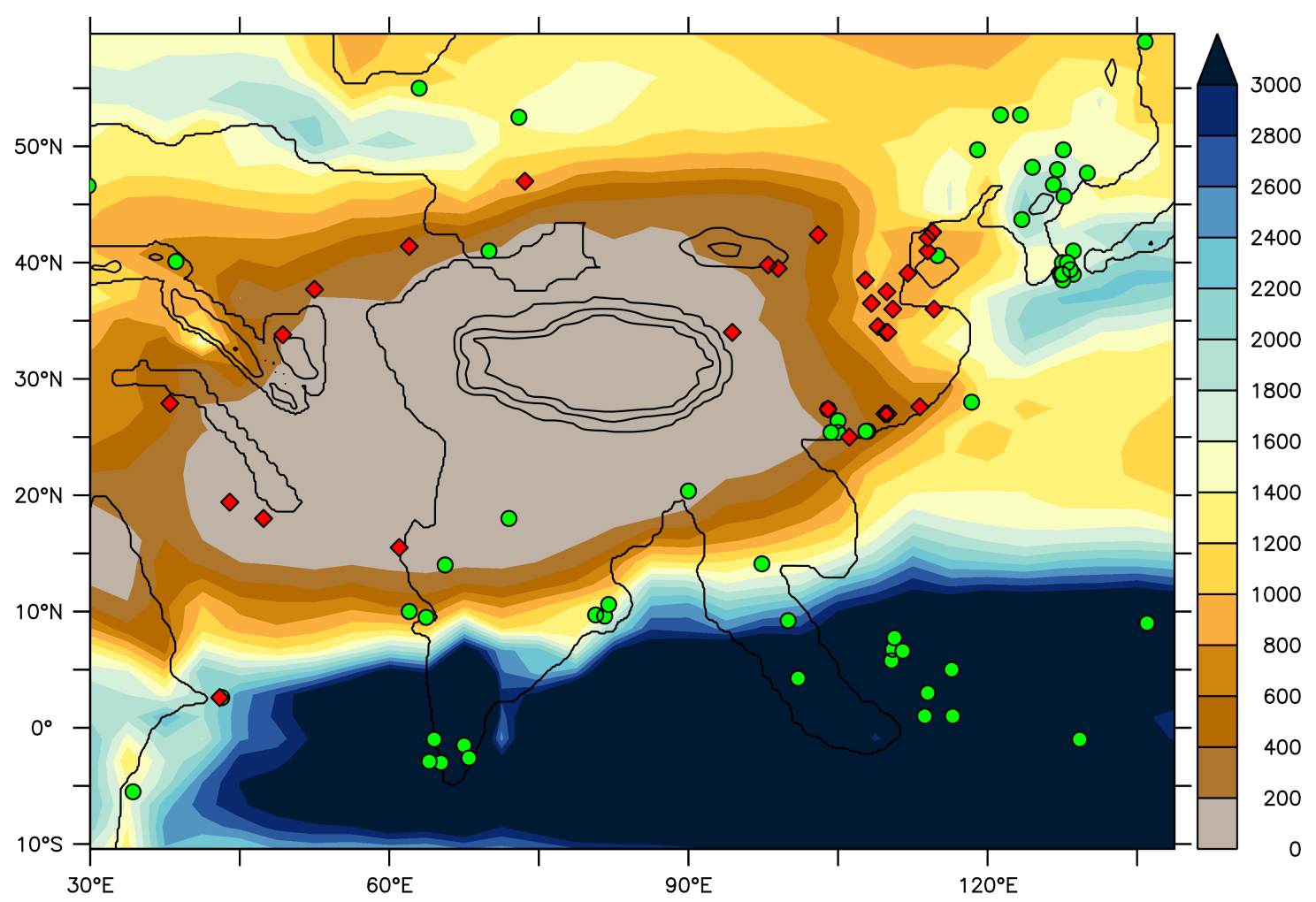




\section{Bibliographical references}

Akhmetiev, M.A., Zaporozhets, N.I., 2014. Paleogene events in Central Eurasia: their role in the flora and vegetation cover evolution, migration of phytochore boundaries, and climate changes. Stratigraphy and Geological Correlation 22, 312-335. https://doi.org/10.1134/S0869593814030022

Baatsen, M., von der Heydt, A.S., Huber, M., Kliphuis, M.A., Bijl, P.K., Sluijs, A., Dijkstra, H.A., 2018. Equilibrium state and sensitivity of the simulated middle-to-late Eocene climate. Climate of the Past Discussions 1-49. https://doi.org/10.5194/cp-2018-43

Boucot, A.J., Xu, C., Scotese, C.R., Morley, R.J., 2013. Phanerozoic Paleoclimate: An Atlas of Lithologic Indicators of Climate. SEPM (Society for Sedimentary Geology), Tulsa, Oklahoma, U.S.A. https://doi.org/10.2110/ sepmcsp. 11

Bougeois, L., Dupont-Nivet, G., de Rafélis, M., Tindall, J.C., Proust, J.-N., Reichart, G.-J., de Nooijer, L.J., Guo, Z., Ormukov, C., 2018. Asian monsoons and aridification response to Paleogene sea retreat and Neogene westerly shielding indicated by seasonality in Paratethys oysters. Earth and Planetary Science Letters 485, 99-110. https://doi.org/10.1016/j.eps1.2017.12.036

Francis, 2007. 100 My of Antarcic climate evolution: Evidence from fossil plants.

Greenwood, D.R., Basinger, J.F., Smith, R.Y., 2010. How wet was the Arctic Eocene rain forest? Estimates of precipitation from Paleogene Arctic macrofloras. Geology 38, 15-18. https://doi.org/10.1130/G30218.1

Herman, A.B., Spicer, R.A., Aleksandrova, G.N., Yang, J., Kodrul, T.M., Maslova, N.P., Spicer, T.E.V., Chen, G., Jin, J.-H., 2017. Eocene-early Oligocene climate and vegetation change in southern China: Evidence from the Maoming Basin. Palaeogeography, Palaeoclimatology, Palaeoecology 479, 126-137. https://doi.org/10.1016/ j.palaeo.2017.04.023

Ho, S.L., Laepple, T., 2016. Flat meridional temperature gradient in the early Eocene in the subsurface rather than surface ocean. Nature Geoscience 9, 606-610. https://doi.org/10.1038/ngeo2763

Hoorn, C., Straathof, J., Abels, H.A., Xu, Y., Utescher, T., Dupont-Nivet, G., 2012. A late Eocene palynological record of climate change and Tibetan Plateau uplift (Xining Basin, China). Palaeogeography, Palaeoclimatology, Palaeoecology 344-345, 16-38. https://doi.org/10.1016/j.palaeo.2012.05.011

Hunt, R.J., 2001. Biodiversity and palaeoecological significance of Tertiary fossil floras from King George Island, West Antarctica. PhD thesis 339.

Kohn, M.J., Strömberg, C.A.E., Madden, R.H., Dunn, R.E., Evans, S., Palacios, A., Carlini, A.A., 2015. Quasi-static Eocene-Oligocene climate in Patagonia promotes slow faunal evolution and mid-Cenozoic global cooling. Palaeogeography, Palaeoclimatology, Palaeoecology 435, 24-37. https://doi.org/10.1016/j.palaeo.2015.05.028

Licht, A., van Cappelle, M., Abels, H.A., Ladant, J.-B., Trabucho-Alexandre, J., France-Lanord, C., Donnadieu, Y., Vandenberghe, J., Rigaudier, T., Lécuyer, C., Terry Jr, D., Adriaens, R., Boura, A., Guo, Z., Soe, A.N., Quade, J., Dupont-Nivet, G., Jaeger, J.-J., 2014. Asian monsoons in a late Eocene greenhouse world. Nature 513, 501-506. https://doi.org/10.1038/nature13704

Ma, X., Jiang, H., Cheng, J., Xu, H., 2012. Spatiotemporal evolution of Paleogene palynoflora in China and its implication for development of the extensional basins in East China. Review of Palaeobotany and Palynology 184, 24-35. https://doi.org/10.1016/j.revpalbo.2012.07.013

Meijer, N., Dupont-Nivet, G., Abels, H.A., Kaya, M.Y., Licht, A., Xiao, M., Zhang, Y., Roperch, P., Poujol, M., Lai, Z., Guo, Z., 2019. Central Asian moisture modulated by proto-Paratethys Sea incursions since the early Eocene. Earth and Planetary Science Letters 510, 73-84. https://doi.org/10.1016/j.epsl.2018.12.031

Morley, R.J., 2018. Assembly and division of the South and South-East Asian flora in relation to tectonics and climate change. Journal of Tropical Ecology 34, 209-234. https://doi.org/10.1017/S0266467418000202 
Saxena, R.K., Trivedi, G.K., n.d. Palynological investigation of the Kopili Formation (Late Eocene) in North Cachar Hills, Assam, India 25.

Sorrel, P., Eymard, I., Leloup, P.-H., Maheo, G., Olivier, N., Sterb, M., Gourbet, L., Wang, G., Jing, W., Lu, H., Li, H., Yadong, X., Zhang, K., Cao, K., Chevalier, M.-L., Replumaz, A., 2017. Wet tropical climate in SE Tibet during the Late Eocene. Scientific Reports 7. https://doi.org/10.1038/s41598-017-07766-9

Spicer, R., Yang, J., Herman, A., Kodrul, T., Aleksandrova, G., Maslova, N., Spicer, T., Ding, L., Xu, Q., Shukla, A., Srivastava, G., Mehrotra, R., Liu, X.-Y., Jin, J.-H., 2017. Paleogene monsoons across India and South China: Drivers of biotic change. Gondwana Research 49, 350-363. https://doi.org/10.1016/j.gr.2017.06.006

Spicer, R.A., Yang, J., Herman, A.B., Kodrul, T., Maslova, N., Spicer, T.E.V., Aleksandrova, G., Jin, J., 2016. Asian Eocene monsoons as revealed by leaf architectural signatures. Earth and Planetary Science Letters 449, 6168. https://doi.org/10.1016/j.eps1.2016.05.036

Taylor, K.W.R., Huber, M., Hollis, C.J., Hernandez-Sanchez, M.T., Pancost, R.D., 2013. Re-evaluating modern and Palaeogene GDGT distributions: Implications for SST reconstructions. Global and Planetary Change 108, 158-174. https://doi.org/10.1016/j.gloplacha.2013.06.011

Utescher, T., Bondarenko, O.V., Mosbrugger, V., 2015. The Cenozoic Cooling - continental signals from the Atlantic and Pacific side of Eurasia. Earth and Planetary Science Letters 415, 121-133. https://doi.org/10.1016/j.epsl. 2015.01.019

Weller, P., Stein, R., 2008. Paleogene biomarker records from the central Arctic Ocean (Integrated Ocean Drilling Program Expedition 302): Organic carbon sources, anoxia, and sea surface temperature: PALEOGENE CENTRAL ARCTIC OCEAN BIOMARKER. Paleoceanography 23, n/a-n/a. https://doi.org/ 10.1029/2007PA001472

Zachos, J., Pagani, M., Sloan, L., Thomas, E., Billups, K., 2001. Trends, Rhythms, and Aberrations in Global Climate 65 Ma to Present. Science 292, 686. https://doi.org/10.1126/science.1059412 